\title{
Complexity and Fermi surface deformation in compressed lithium
}

\author{
A. Rodriguez-Prieto, ${ }^{1,2}$ A. Bergara, ${ }^{1,2,3}$ V. M. Silkin, ${ }^{2}$ and P. M. Echenique ${ }^{2,3,4}$ \\ ${ }^{1}$ Materia Kondentsatuaren Fisika Saila, Zientzi Fakultatea, Euskal Herriko Unibertsitatea, 644 Posta kutxatila, E-48080 Bilbo, \\ Basque Country, Spain \\ ${ }^{2}$ Donostia International Physics Center (DIPC), Manuel de Lardizabal Pasealekua, E-20018 Donostia, Basque Country, Spain \\ ${ }^{3}$ Centro Mixto CSIC-UPV/EHU, 1072 Posta kutxatila, E-20080 Donostia, Basque Country, Spain \\ ${ }^{4}$ Materialen Fisika Saila, Kimika Fakultatea, Euskal Herriko Unibertsitatea, 1072 Posta kutxatila, E-20080 Donostia, \\ Basque Country, Spain
}

(Received 29 June 2006; published 14 November 2006)

\begin{abstract}
Recently reported structural complexity and enhanced temperature superconducting transitions in lithium under pressure have increased the interest in light alkalies, otherwise considered as simple and well-known systems under normal conditions. Here we present an analysis of the pressure-induced Fermi surface deformation in lithium and its relation to the observed complexity. According to our calculations, the Fermi surface becomes increasingly anisotropic with pressure and at $8 \mathrm{GPa}$ contacts the Brillouin zone boundary inducing a Hume-Rothery mechanism explaining the bcc-fcc transition. Around $30 \mathrm{GPa}$ increasing cooper-like necks and an extended nesting are observed in the Fermi surface in the fcc phase, enhancing the electronic susceptibility response function and inducing a strong phonon softening. This softening, besides preluding the transition to complex structures and providing a better understanding of the observed superconductivity, is expected to induce other yet unexplored anomalies in compressed lithium.
\end{abstract}

\section{DOI: 10.1103/PhysRevB.74.172104}

Light alkali metals have usually been considered as simple metals due to their monovalency, high conductivity, and crystallization in high-symmetric structures. Under normal conditions the interaction between valence electrons and ionic cores is weak in these elements and the simple nearly free electron (NFE) model has been considered a good approximation to analyze their properties, ${ }^{1}$ which can be easily checked by their almost spherical Fermi surfaces (FS's). At normal conditions of pressure and temperature, lithium adopts a bcc structure, and at around $8 \mathrm{GPa}$ the compact fcc phase starts to be favored. However, recently both theoretical and experimental results have radically changed our picture of the alkali metals as simple systems, as phase transitions to complex and low-coordinated structures have been reported to emerge. ${ }^{2-5} \mathrm{~A}$ recent $\mathrm{x}$-ray analysis ${ }^{3}$ revealed that lithium undergoes a fcc to a rombohedral $h R 1$ transition at $39 \mathrm{GPa}$ and a $h R 1$ to $c I 16$ (with 16 atoms per unit cell) at $41 \mathrm{GPa}$. Complex structural transitions under pressure are not unique in lithium, but have been predicted and observed in heavier alkali metals as well. ${ }^{6-11}$ Surprisingly, recent experiments have shown that, despite the increasing resistivity with pressure, ${ }^{12,13}$ lithium superconducts at around $20 \mathrm{~K}$ when the applied pressure rises to $30 \mathrm{GPa},{ }^{14-16}$ becoming the highest superconducting transition temperature between simple elements. ${ }^{17}$ It is noteworthy that experiments looking for superconductivity in lithium under ambient pressure have failed, ${ }^{18}$ which even has raised interest in characterizing the physical properties of compressed lithium.

In this article we present the evolution of the Fermi surface topology in lithium under pressure as a key ingredient determining the modification of its physical properties. We find that increasing pressure leads to a strong anisotropic deformation of its Fermi surface. ${ }^{19}$ Among other features discussed below, at $8 \mathrm{GPa}$ it touches the Brillouin zone (BZ) boundary so that a Hume-Rothery mechanism can explain the observed bcc-fcc transition. At around $30 \mathrm{GPa}$ an extended nesting develops along the fcc $\Gamma W$ direction which
PACS number(s): 71.18.+y, 71.20.Dg, 74.25.Kc, 74.62.Fj

profoundly modifies its electronic properties. As a result of the high number of electronic states coupled by the nesting momentum, the static electronic response changes and induces a phonon softening, which increases the electronphonon interaction needed to understand ${ }^{20-22}$ the observed high superconducting transition temperature.

In order to analyze the electronic properties of lithium under pressure we have used a plane-wave implementation of density functional theory (DFT) within the local density approximation (LDA), as implemented in the Vienna ab initio simulation package (VASP). ${ }^{23,24}$ Although at high pressure an important core overlap is observed, we have checked that the projector augmented-wave (PAW) approximation, ${ }^{25}$ which fully treats all the electrons, and ultrasoft pseudopotentials ${ }^{26}$ give similar results. Figures of threedimensional Fermi surfaces have been generated by XCRYSDEN $^{27}$ and phonon frequencies have been computed with density functional perturbation theory (DFPT), as implemented in the plane-wave self-consistent field (PWSCF) code. $^{28}$ Convergence is achieved with a Monkhorst-Pack mesh of $20 \times 20 \times 20$ and an energy cutoff of $430 \mathrm{eV}$.

An essential feature determining electronic properties of any metal is the topology of the Fermi surface. Figure 1 shows the Fermi surface of lithium at three selected pressures. As a result of the increasing nonlocal character of the pseudopotential, ${ }^{29,30}$ a significant $s-p$ mixing is induced and its spheroidal free-electron-like shape at normal conditions deforms significantly under compression. Although at equilibrium there is no BZ-FS contact, at $8 \mathrm{GPa}$ the FS starts touching the BZ at the $N$ point. This fact leads to the observed bec-fcc structural transition, which can be understood via the so-called Hume-Rothery effect. The interaction between the BZ boundary and the FS develops extended necks along the fcc $\Gamma L$ direction where a pseudogap opens and, therefore, reduces the overall electronic energy. Figure 2 shows the phonon frequencies of bcc lithium at equilibrium 

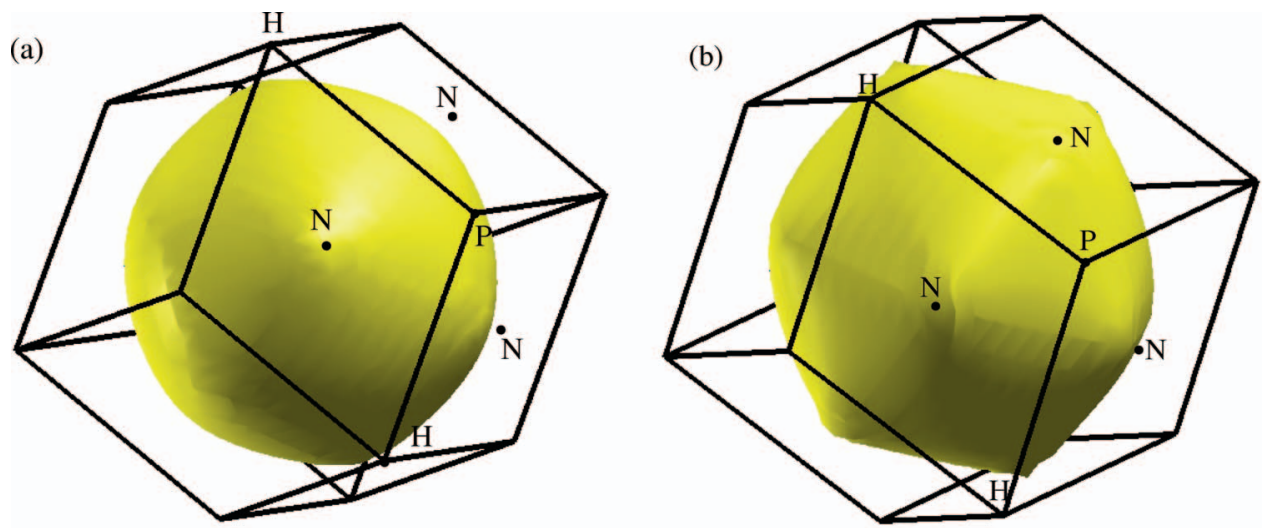

FIG. 1. (Color) Fermi surface of bec lithium at (a) equilibrium and (b) $P=8 \mathrm{GPa}$ and $\mathrm{fcc}$ at (c) $P=30 \mathrm{GPa}$. The spheroidal Fermi surface at equilibrium becomes increasingly distorted under pressure and at $8 \mathrm{GPa}$, corresponding to the experimentally observed bcc-fcc transition, contacts the

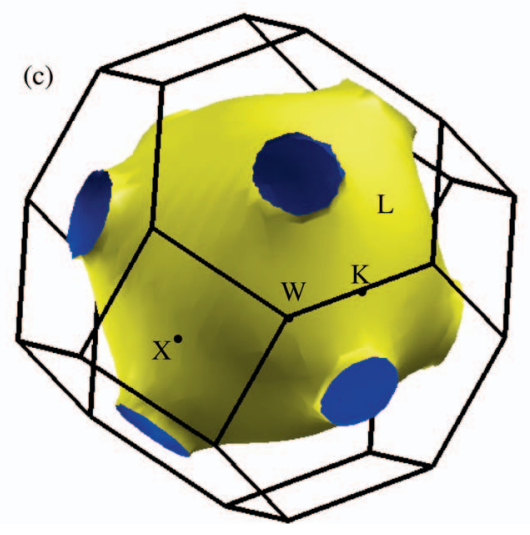
Brillouin zone boundary at the bcc $N$ point. At $P=30 \mathrm{GPa}$ the Fermi surface of fcc lithium shows increasing cooper-like necks along $\Gamma L$ and, more interestingly, develops an extended nesting along several directions, which becomes the origin of profound modifications on its physical properties.

and $8 \mathrm{GPa}$. While phonon frequencies of longitudinal modes monotonically increase with pressure, when pressure is increased to $8 \mathrm{GPa}$ the whole $T_{1}$ branch becomes unstable along the $\Gamma N$ direction and the tetragonal shear elastic constant becomes negative. Actually, at this pressure the bcc phase also has a higher enthalpy than the fcc one, which makes the bcc structure both dynamically and thermodynamically unstable and explains the experimentally observed bec-fcc structural transition.

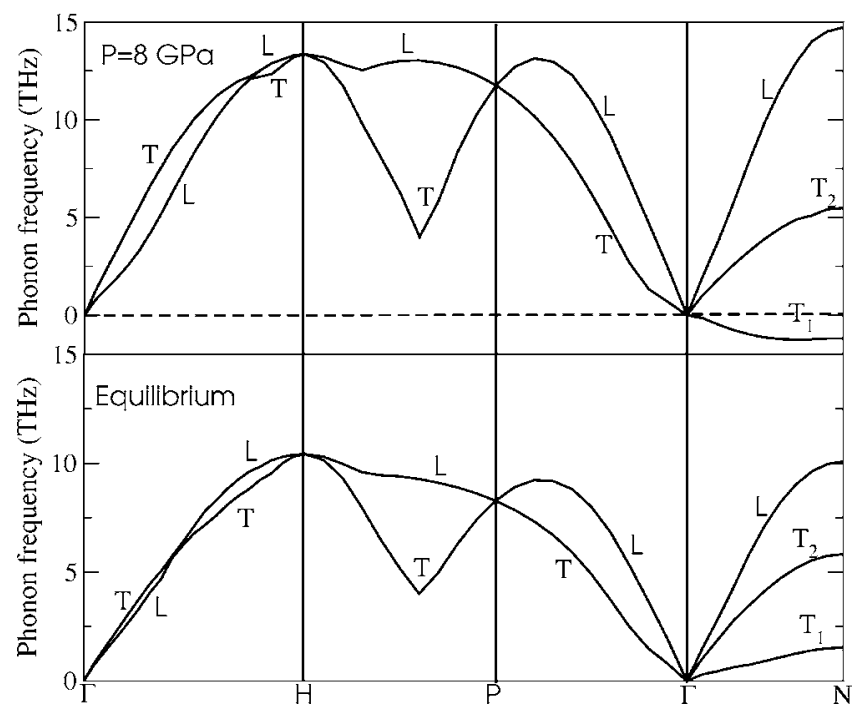

FIG. 2. Phonon frequencies of bcc lithium at equilibrium and $P=8 \mathrm{GPa}$. $L$ and $T$ label longitudinal and transverse modes, respectively. It is remarkable that at $P=8 \mathrm{GPa}$ the whole $T_{1}$ branch becomes unstable along the $\Gamma N$ direction.
At around $30 \mathrm{GPa}$ in the fcc phase an extended nesting on the Fermi surface along different directions is observed. Figure 3 displays several Fermi surface cross sections at $30 \mathrm{GPa}$. Although the cross section along the $\Gamma K X$ plane shows an almost perfect circle, it becomes highly distorted in the $\Gamma K L$ and $\Gamma W X$ planes, with necks along $\Gamma L$ and a welldefined nesting in the $\Gamma K$ and $\Gamma W$ directions. A very similar distortion has also been predicted in a compressed lithium monolayer, ${ }^{5}$ where the free-electron-like circular Fermi line at equilibrium surprisingly becomes a perfect square under pressure, as corresponds to a half-filled tight-binding-like model appropriate to describe localized electronic states.

In order to analyze the influence of this nesting on its physical properties, we have calculated diagonal contributions to the $a b$ initio static electronic susceptibility matrix of the noninteracting electron system:

$$
\begin{aligned}
\chi^{0}(\mathbf{q})= & \frac{1}{\Omega} \sum_{\mathbf{k}}^{B Z} \sum_{n, n^{\prime}} \frac{f_{n \mathbf{k}}-f_{n^{\prime} \mathbf{k}+\mathbf{q}}}{\varepsilon_{n \mathbf{k}}-\varepsilon_{n^{\prime} \mathbf{k}+\mathbf{q}}} \times\left\langle\psi_{n \mathbf{k}}\left|e^{-\mathrm{i} \mathbf{q} \cdot \mathbf{r}}\right| \psi_{n^{\prime} \mathbf{k}+\mathbf{q}}\right\rangle \\
& \times\left\langle\psi_{n^{\prime} \mathbf{k}+\mathbf{q}}\left|e^{\mathbf{i q} \cdot \mathbf{r}}\right| \psi_{n \mathbf{k}}\right\rangle .
\end{aligned}
$$

Here $\mathbf{k}$ is wave vector in the first $\mathrm{BZ}, f_{n \mathbf{k}}$ represents the Fermi-Dirac distribution function, $\Omega$ is the normalization volume, and if $\mathbf{k}+\mathbf{q}$ lies outside the first $\mathrm{BZ}$, it is translated back in by addition of the appropiate reciprocal-lattice vector. The first summation in Eq. (1) was performed with the use of $96 \times 96 \times 96 \mathbf{k}$ mesh, which correspond to 442368 points in the BZ. The second sum runs over the occupied and all unoccupied valence bands up to an energy of $50 \mathrm{eV}$ above Fermi level. 

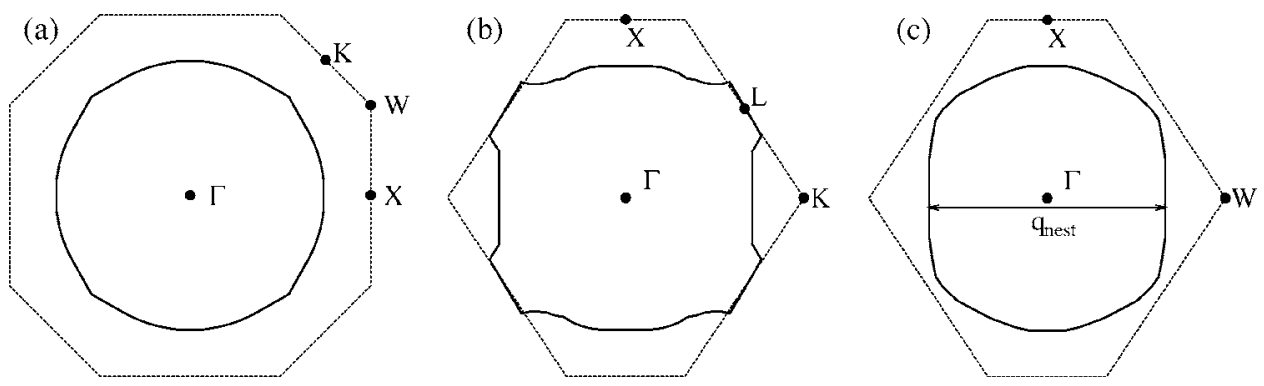

FIG. 3. Fermi surface cross sections (solid lines) of fcc lithium at $30 \mathrm{GPa}$ along the (a) $\Gamma K X$ (100), (b) $\Gamma K L$ (110), and (c) $\Gamma W X(210)$ planes. The Brillouin zone boundary is represented by dotted lines. The cross section along the $\Gamma K X$ plane shows an almost perfect circle whereas in both the $\Gamma K L$ and $\Gamma W X$ planes present a clear nesting in the $\Gamma K$ and $\Gamma W$ directions, respectively.

Although it has been previously reported ${ }^{19,21,22}$ that the nesting along the $\Gamma K$ direction has the strongest influence, we have not seen any special feature on the static susceptibility along this direction. However, an interesting kink emerges at $\mathbf{q}_{\text {nest }}=2 \pi / a(4 / 3,2 / 3,0)\left(\approx 2 k_{\mathrm{F}}\right)$ corresponding to the observed nesting along $\Gamma W$ [see Fig. 3(c)]. Although this nesting in the $\Gamma W X$ plane also emerges at lower pressures, for elevated pressure the nesting is extended to parallel planes as well, growing the nested Fermi surface area. Therefore, as can be seen in the evolution under pressure of the peak in the electronic susceptibily [Fig. 4(b)], the number of electronic states coupled by the nesting momentum vector increases under pressure.

Figure 5 displays the phonon frequencies of fcc lithium along some high-symmetry directions at two selected pressures, 10 and $30 \mathrm{GPa}$. While longitudinal phonon frequencies increase with pressure, a remarkable softening is observed in the lowest transverse mode $\left(T_{1}\right)$, especially along $\Gamma K$ where it becomes unstable at $\mathbf{q}_{\text {inst }}=2 \pi / a(-2 / 3,2 / 3,0)$. Interestingly, this instability is connected to the kink in the electronic susceptibility at $\mathbf{q}_{\text {nest }}$. In order to compare both momenta we have to take into account that $\mathbf{q}_{\text {nest }}$ extends out from the first BZ, which after folding back with $\mathbf{G}=2 \pi / a(-2,0,0)$ results in being $\mathbf{q}_{\text {inst }}, \mathbf{q}_{\text {inst }}=\mathbf{q}_{\text {nest }}+\mathbf{G}$. However, experiments show that fcc lithium is stable up to around $40 \mathrm{GPa}$, indicating that, despite the softening, the observed instability within the harmonic approximation could be stabilized to even higher pressures by anharmonic effects. This softening induced by a strong electron-phonon interaction corresponding to the Kohn anomaly associated with the nesting in the FS, besides preceding the transition to complex structures, provides a better understanding of the superconducting transition ${ }^{20-22}$ observed in this pressure range. It is also interesting to remark that according to Fig. 4(a) the screening corresponding to the $a b$ initio calculation becomes lower than in the free electron approximation. Therefore, band structure effects are expected to increase the repulsive character of the Coulomb pseudopotential $\mu^{*}$ needed to evaluate $T_{c}$. The principal reason for this difference corresponds to the lowering under pressure of the occupied bandwidth ${ }^{2,30}$ and the opening of a pseudogap at $N$ (necks in the FS). Both features are associated with the increasing nonlocal effects ${ }^{30}$ under pressure and will increase the energy difference between occupied and unoccupied electronic states in the denominator of Eq. (1) and, therefore, decrease the screening contribution under pressure.

In conclusion, we have performed ab initio calculations of the Fermi surface deformation with increasing pressure in bulk lithium. Although the nearly free electron model correctly describes the properties of lithium at normal conditions, it loses its validity as the electronic density increases. As a result of the increasing nonlocal character of the pseudopotential, the Fermi surface of bcc lithium becomes

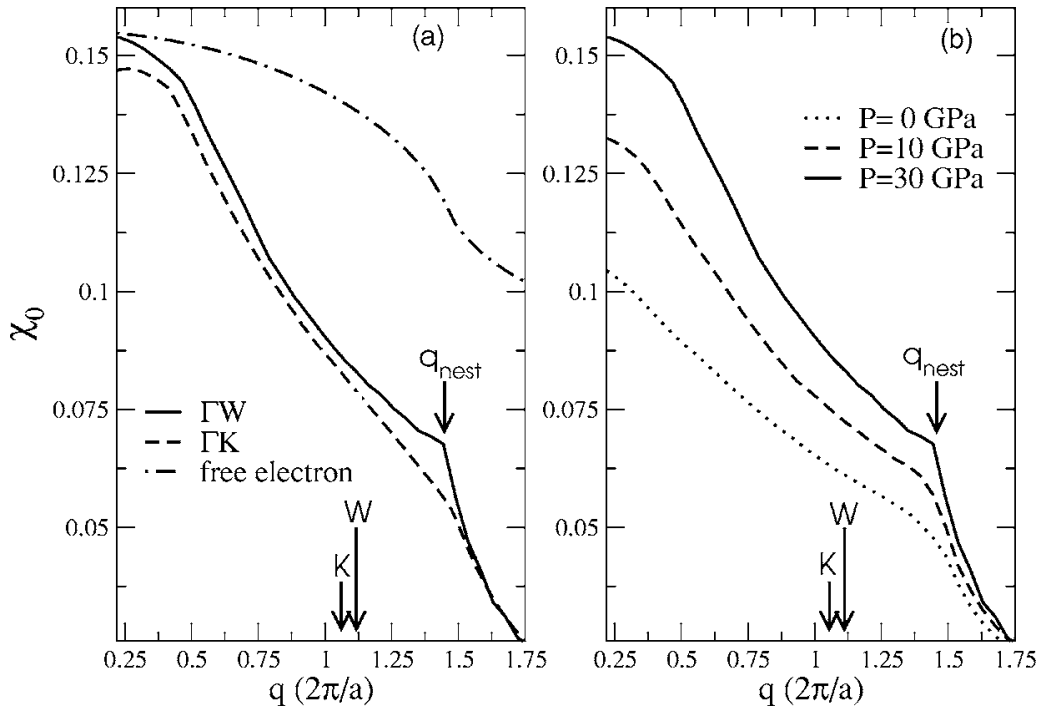

FIG. 4. (a) Static ab initio susceptibility along $\Gamma K$ (dashed line) and $\Gamma W$ (solid line) directions at $P=30 \mathrm{GPa}$ and (dash-dotted line) the free electron approximation (Lindhard function) calculated with the $a b$ initio effective mass $m^{*}=2 m_{e}$. For clarity, the locations of $W$ and $K$ points in the $\mathrm{BZ}$ are shown in the figure and, although no special feature is seen along $\Gamma K$, a kink emerges along $\Gamma W$ at the nesting momentum $\left(\mathbf{q}_{\text {nest }}\right)$, which extends out from the first BZ. Band effects are shown to decrease the electronic screening compared to the free electron approximation. (b) The evolution under pressure of the kink observed in the $a b$ initio susceptibility along the $\Gamma W$ direction. 


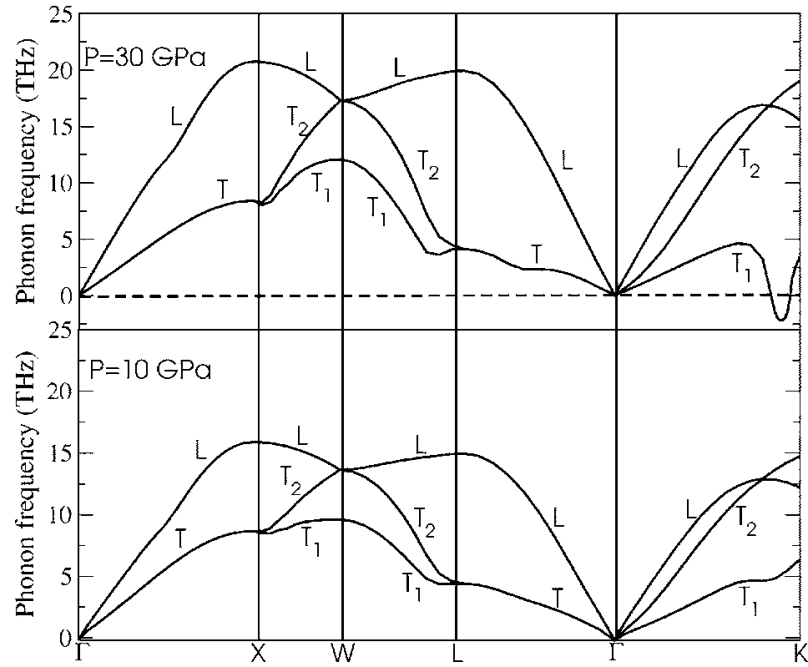

FIG. 5. Phonon frequencies of fcc lithium at $P=10 \mathrm{GPa}$ and $P=30 \mathrm{GPa}$. Besides the general softening of the transverse $T_{1}$ branch, the well-defined instability of the $T_{1}$ branch along $\Gamma K$ with the momentum connected to the kink in the electronic susceptibility is remarkable, which is associated with the nesting observed in the Fermi surface.

highly anisotropic under pressure and at $8 \mathrm{GPa}$ starts touching the BZ at the bcc $N$ point, which allows us to explain the observed bcc-fcc transition via a Hume-Rothery mechanism.
Furthermore, as a result of the increasing electronic localization, at $30 \mathrm{GPa}$ the Fermi surface of fcc lithium even shows an extended nesting along the $\Gamma W$ direction which modifies its electronic properties and induces a phonon instability associated with the nesting. This softening and the anisotropic deformation of the Fermi surface, besides preceding the transition to complex structures and providing a better understanding of the observed superconducting transition, is expected to induce other yet unexplored anomalies in compressed lithium. For example, dropping bands below the Fermi energy will enhance electronic interband excitations and, therefore, profoundly modify its dynamic and optical properties. ${ }^{31}$ It is noteworthy that we also expect interesting anomalies in the melting curve of lithium, similar to those seen in sodium, ${ }^{32}$ as the observed general softening of transverse phonon modes under pressure might induce a dramatic lowering of the thermal energy required to melt compressed lithium. ${ }^{33}$

The authors thank N.W. Ashcroft for the very useful and stimulating discussions. We acknowledge partial support by the University of the Basque Country, the Basque Herkuntza, Unibersitate eta Ikerketa saila, and Spanish MCyT (Grant No. FIS 2004-06490-C03-01), and the European Community 6th Network of Excellence NANOQUANTA (Grant No. NMP4-CT-2004-500198).
${ }^{1}$ E. Wigner and F. Seitz, Phys. Rev. 43, 804 (1933).

${ }^{2}$ J. B. Neaton and N. W. Ashcroft, Nature (London) 400, 141 (1999).

${ }^{3}$ M. Hanfland, K. Syassen, N. E. Christensen, and D. L. Novikov, Nature (London) 408, 174 (2000).

${ }^{4}$ N. E. Christensen and D. L. Novikov, Phys. Rev. Lett. 86, 1861 (2001).

${ }^{5}$ A. Rodriguez-Prieto and A. Bergara, Phys. Rev. B 72, 125406 (2005).

${ }^{6}$ J. B. Neaton and N. W. Ashcroft, Phys. Rev. Lett. 86, 2830 (2001).

${ }^{7}$ M. Hanfland, I. Loa, and K. Syassen, Phys. Rev. B 65, 184109 (2002).

${ }^{8}$ M. I. McMahon, S. Rekhi, and R. J. Nelmes, Phys. Rev. Lett. 87, 055501 (2001).

${ }^{9}$ M. I. McMahon, R. J. Nelmes, and S. Rekhi, Phys. Rev. Lett. 87, 255502 (2001).

${ }^{10}$ R. J. Nelmes, M. I. McMahon, J. S. Loveday, and S. Rekhi, Phys. Rev. Lett. 88, 155503 (2002).

${ }^{11}$ N. E. Christensen and D. L. Novikov, Solid State Commun. 119, 477 (2001)

${ }^{12}$ V. E. Fortov, V. V. Yakushev, K. L. Kagan, I. V. Lomonosov, V. I. Postnov, T. I. Yakusheva, and A. N. Kuryanchik, JETP Lett. 74, 418 (2001).

${ }^{13}$ K. Shimizu, H. Ishikawa, and K. Amaya, J. Phys.: Condens. Matter 14, 10433 (2002).

${ }^{14}$ K. Shimizu, H. Ishikawa, D. Takao, T. Yagi, and K. Amaya, Nature (London) 419, 597 (2002).

${ }^{15}$ V. V. Struzhkin, M. I. Eremets, W. Gan, H. K. Mao, and R. J. Hemley, Science 298, 1213 (2002).
${ }^{16}$ S. Deemyad and J. S. Schilling, Phys. Rev. Lett. 91, 167001 (2003).

${ }^{17}$ N. W. Ashcroft, Nature (London) 419, 569 (2002).

${ }^{18}$ K. I. Juntunen and J. T. Tuoriniemi, Phys. Rev. Lett. 93, 157201 (2004).

${ }^{19}$ A. Rodriguez-Prieto and A. Bergara, cond-mat/0505619 (unpublished).

${ }^{20}$ J. S. Tse, Y. Ma, and H. M. Tutuncu, J. Phys.: Condens. Matter 17, S911 (2005).

${ }^{21}$ D. Kasinathan, J. Kunes, A. Lazicki, H. Rosner, C. S. Yoo, R. T. Scalettar, and W. E. Pickett, Phys. Rev. Lett. 96, 047004 (2006).

${ }^{22}$ G. Profeta, C. Franchini, N. N. Lathiotakis, A. Floris, A. Sanna, M. A. L. Marques, M. Luders, S. Massidda, E. K. U. Gross, and A. Continenza, Phys. Rev. Lett. 96, 047007 (2006).

${ }^{23}$ G. Kresse and J. Hafner, Phys. Rev. B 47, 558 (1993).

${ }^{24}$ G. Kresse and J. Furthmüller, Phys. Rev. B 54, 11169 (1996).

${ }^{25}$ P. E. Blöchl, Phys. Rev. B 50, 17953 (1994).

${ }^{26}$ D. Vanderbilt, Phys. Rev. B 41, 7892 (1990).

${ }^{27}$ A. Kokalj, J. Mol. Graphics 17, 176 (1999).

${ }^{28}$ S. Baroni, A. del Corso, S. de Gironcoli, and P. Gianozzi, http:// www.pwscf. org

${ }^{29}$ J. C. Boettger and S. B. Trickey, Phys. Rev. B 32, 3391 (1985).

${ }^{30}$ A. Bergara, J. B. Neaton, and N. W. Ashcroft, Phys. Rev. B 62, 8494 (2000).

${ }^{31}$ V. M. Silkin, A. Rodriguez-Prieto, A. Bergara, A. V. Chulkov, and P. M. Echenique (unpublished).

${ }^{32}$ E. Gregoryanz, O. Degtyareva, M. Somayazulu, R. J. Hemley, and H. K. Mao, Phys. Rev. Lett. 94, 185502 (2005).

${ }^{33}$ A. Rodriguez-Prieto, D. Alfe, and A. Bergara (unpublished). 